Please do not remove this page

RMIT

UNIVERSITY

\title{
GPS satellite velocity and acceleration determination using the broadcast ephemeris
}

Zhang, Jason; Zhang, Kefei; Grenfell, Ronald; Deakin, Rodney

https://researchrepository.rmit.edu.au/esploro/outputs/9921859559201341/filesAndLinks?institution=61RMIT_INST\&index=null

Zhang, J., Zhang, K., Grenfell, R., \& Deakin, R. (2006). GPS satellite velocity and acceleration determination using the broadcast ephemeris. Journal of Navigation, 59, 293-305.

https://doi.org/10.1017/s0373463306003638

Published Version: https://doi.org/10.1017/s0373463306003638

Repository homepage: https://researchrepository.rmit.edu.au

Copyright (c) 2006 The Royal Institute of Navigation

Downloaded On 2023/04/26 11:43:24 +1000 


\title{
GPS Satellite Velocity and Acceleration Determination using the Broadcast Ephemeris
}

\author{
Jason Zhang ${ }^{1}$, Kefei Zhang, ${ }^{1,2}$, Ron Grenfell ${ }^{1}$, Rod Deakin ${ }^{1}$ \\ ${ }^{1}$ (RMIT University Melbourne) \\ (Email: kefei.zhang@rmit.edu.au) \\ ${ }^{2}$ (China University of Mining Technology, Xuzhou)
}

Satellite velocity determination using the broadcast ephemeris is discussed and it is pointed out that the conventional rotation matrix method involves a complicated process of computation. This paper proposes an alternative method using a simple differentiator to derive satellite Earth-Centred-Earth-Fixed (ECEF) velocity from the ECEF satellite positions that are calculated using the standard ICD-GPS-200 algorithm. The proposed algorithm simplifies the velocity transformation procedure, and therefore provides a good alternative. It is demonstrated that $\pm 1 \mathrm{~mm} / \mathrm{s}$ per axis ECEF satellite velocity is achievable by using the first-order central difference of a Taylor series approximation.

A closed-form formula is also derived for the determination of GPS satellite ECEF acceleration using the broadcast ephemeris. This formula is capable of accuracies better than $\pm 0 \cdot 1 \mathrm{~mm}$ per second squared in each axis. With such a high accuracy of satellite acceleration in real-time, it is possible to detect the line-of-sight range acceleration precisely and as such it is concluded that a GPS receiver can be considered as a precise accelerometer.

The success of the position differential method implies that real-time satellite ECEF velocities can be directly derived through numerical differentiation of the position polynomials. This is desirable for GPS velocity determination applications which require high output rate results in real-time. The derived closed-form formula for GPS satellite ECEF acceleration would benefit those who attempt to use a GPS receiver as an accurate accelerometer in real-time.

\section{KEY WORDS \\ 1. GPS velocity. 2. GPS acceleration. 3. Broadcast Ephemeris.}

1. INTRODUCTION. GPS can be used in precise velocity and acceleration determination applications as varied as automobile brake system testing, athlete monitoring, airborne gravimetry and GPS/INS integration. In such applications, a GPS satellite is a moving signal source in space, from which the relative motion between the satellite and a receiver is sensed in terms of the Doppler shift and its change rate. The velocities and accelerations of at least four GPS satellites must be determined and taken as known values before velocity and acceleration of a user can be determined.

The principle of velocity determination using GPS Doppler measurements has been presented by e.g. Misra and Enge (2001) and Hofmann-Wellenhof et al. (2001). 
Acceleration determination using GPS Doppler rates is proposed by Kleusberg et al. (1990), followed by Jekeli (1994) and Jekeli and Carcia (1997). With the Selective Availability (SA) turned off and the advancement of GPS receiver technology, it is now possible for a user to get his/her precise velocity in sub-centimetre per second level in a standalone mode and in real-time (Simsky and Boon, 2003, Zhang et al., 2004). However, it is reported that real-time acceleration determination may be affected by the accuracy of the satellite acceleration estimation using the broadcast ephemeris (Kennedy, 2003).

The GPS orbit parameters are formatted according to the user Interface Control Document ICD-GPS-200c (ARINC, 2000), wherein the satellite Earth-CentredEarth-Fixed (ECEF) position algorithms are well presented. However the calculation of satellite ECEF velocity and acceleration using the broadcast ephemeris is not described in the ICD-GPS-200c. This paper discusses how to get satellite ECEF velocity and acceleration using the GPS broadcast ephemeris. It briefly describes the "natural satellite" orbital system, and addresses its difference from the ICDGPS-200c orbital system. The main differences between the two systems affect the way that the broadcast ephemeris is constructed (e.g. broadcast parameterisation) and the consequent changes to the coordinate transform procedures. For instance, there is no change rate of the argument of perigee in the GPS navigation message. Although the ICD-GPS-200c scheme can effectively represent a satellite's positions in its short arc orbit whilst reducing the payload of satellite navigation message, it is rather complicated to transform orbital satellite velocities to the ECEF system when the rotation matrix method is adopted.

A simple alternative method is presented, which uses the first-order central difference of a Taylor series approximation of the satellite ECEF positions. The velocity through ECEF position differentiation is numerically identical to the velocity obtained through the rotation matrix method and close to the precise velocity from the International GPS Service (IGS) SP3 precise ephemeris (Spofford and Remondi, 2004) at $\pm 1 \mathrm{~mm} / \mathrm{s}$ level.

A closed-form ECEF satellite acceleration formula is also derived by the rotation matrix method. It is demonstrated that the discrepancies of the accelerations derived from the rotation matrix method and the IGS SP3 precise ephemeris are within $\pm 0 \cdot 1 \mathrm{~mm} / \mathrm{s}^{2}$ per axis. It is concluded that the proposed algorithms for GPS satellite ECEF velocity and acceleration determination can achieve accuracies of millimetres per second $(\mathrm{mm} / \mathrm{s})$ and millimetres per second squared $\left(\mathrm{mm} / \mathrm{s}^{2}\right)$ respectively in real-time.

\section{SATELLITE ORBIT REPRESENTATION BY KEPLERIAN} PARAMETERS. A satellite orbiting the Earth follows Keplerian laws. However, due to the Earth's non-central gravity field, attractions from the Sun, Moon and other disturbing forces, there are perturbations in the GPS orbit. Figure 1 illustrates the Keplerian orbital elements and a satellite position. The satellite's position in an ideal, non-perturbed orbit can be represented by:

- size and shape of the ellipse: semi-major axis $a$ and eccentricity $e$;

- orientation of the orbital plane relative to the Earth: orbit inclination $I$ and longitude of the ascending node $\Omega$; 


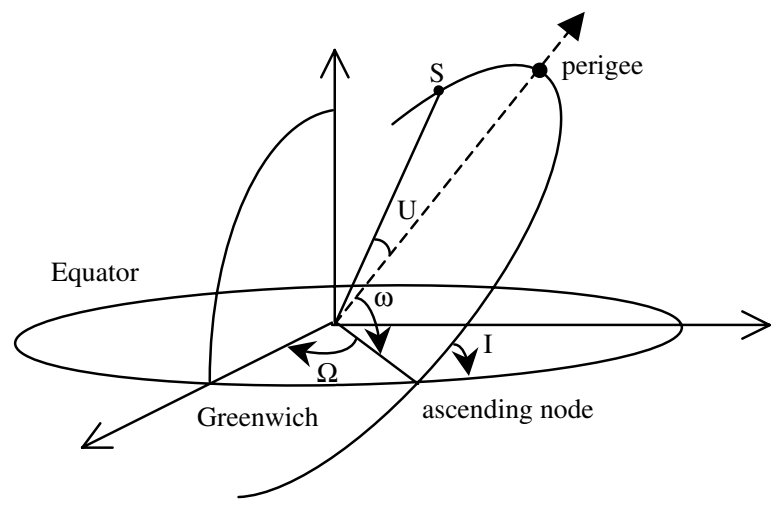

Figure 1. Keplerian elements and satellite orbit.

- orientation of the ellipse in the orbital plane: argument of perigee $\omega$;

- satellite position in the ellipse: true anomaly $U$; and

- a reference time: $t$ (time passes the perigee), or $t_{o e}$ (time of the reference ephemeris)

2.1. Satellite position, velocity and acceleration in the "natural" orbital plane. The "natural" satellite orbital plane system is defined by the origin located at one focus of the elliptical orbit, which corresponds to the position of the mass centre of the Earth. The x-axis and y-axis are coincident with the major and minor axes of the orbital ellipse respectively. Figure 2 illustrates the natural orbital plane system where the positive $\mathrm{x}$-axis passes through perigee.

A satellite position in the natural orbital plane system can be expressed (Beutler, 1998, Misra and Enge, 2001) by

$$
\vec{r}=\left(\begin{array}{c}
a \cos E-a \cdot e \\
a \sqrt{1-e^{2}} \sin E \\
0
\end{array}\right)=\left(\begin{array}{c}
r \cos U \\
r \sin U \\
0
\end{array}\right)=\left(\begin{array}{l}
x \\
y \\
0
\end{array}\right)
$$

where: $a$ is the semi-major axis of the satellite orbit; $e$ is the eccentricity of the orbit; $E$ is the orbital eccentric anomaly; $r$ is the instantaneous distance between the satellite and the centre of the Earth; and $U$ is the true anomaly.

A satellite velocity in the natural orbital plane system is given by (ibid)

$$
\dot{\vec{r}}=\frac{a \cdot n}{1-e \cos E} \cdot\left(\begin{array}{c}
-\sin E \\
\sqrt{1-e^{2}} \cos E \\
0
\end{array}\right)
$$

where $n$ is the mean motion of the satellite. The satellite acceleration in the orbital plane system is (ibid)

$$
\ddot{\vec{r}}=-\frac{G M}{r^{3}} \cdot \vec{r}
$$

where $G$ is the universal gravitational constant and $M$ is the Earth's mass. 


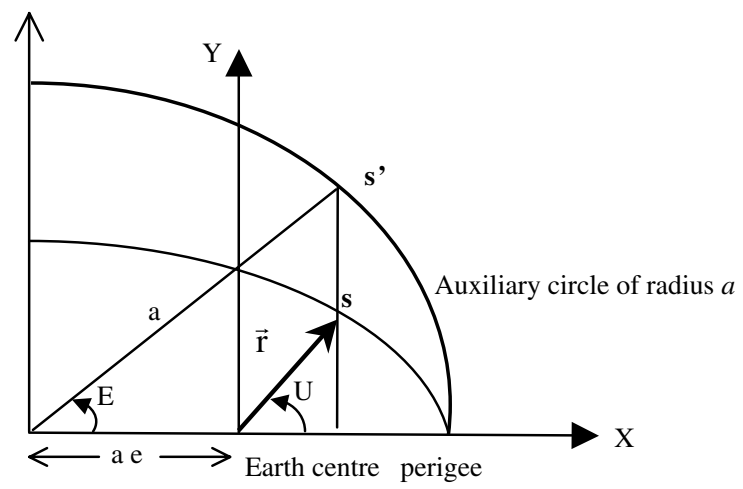

Figure 2. Satellite position in the orbital plane coordinate system.

From Figure 1, it can be seen that the transformation of the Cartesian components of a satellite position vector $\vec{r}$ from the orbital plane coordinate system to the ECEF system may be carried out by three rotations in the following order:

- a first rotation by the argument of the perigee $\omega$;

- a second rotation by the angle of inclination $I$; and

- finally a rotation by the angle of the longitude of ascending node $\Omega$.

The corresponding transform equation is

$$
\vec{r}_{E C E F}=R_{3}(-\Omega) \cdot R_{1}(-I) \cdot R_{3}(-\omega) \vec{r}
$$

where $R_{n}(\theta)$ is the rotation matrix, the subscript $n=1,3$ corresponding to the rotation axes of $x, z$ respectively. The rotation matrixes $R_{3},(\theta) R_{1}(\theta)$ are expressed in the forms of (cf., Farrell and Barth, 1999, p.34)

$$
R_{1}(\theta)=\left[\begin{array}{ccc}
1 & 0 & 0 \\
0 & \cos \theta & \sin \theta \\
0 & -\sin \theta & \cos \theta
\end{array}\right] \quad R_{3}(\theta)=\left[\begin{array}{ccc}
\cos \theta & \sin \theta & 0 \\
-\sin \theta & \cos \theta & 0 \\
0 & 0 & 1
\end{array}\right]
$$

2.2. ICD-200 orbital coordinate system. The user algorithm for GPS satellite ECEF position determination from the broadcast ephemeris has been well documented in the ICD-GPS-200c. It is important to stress that the orbital coordinate system used in the ICD-GPS-200c (ICDorb) is different from the above "natural" orbital system. The ICDorb system has the positive $\mathrm{x}$-axis passing through the ascending node of the orbital plane rather than perigee. The difference is reflected mainly in the parameterisation and calculation of the three pairs of amplitudes of harmonic correction terms for the sinusoid correction models of the argument of latitude, orbit radius and inclination angle, see Equations (16) and (17). By such a coordinate system definition, one only requires two rotations to transform a position from the orbital system to the ECEF system, i.e.

$$
\vec{r}_{E C E F}=R_{3}\left(-\Omega_{c}\right) \cdot R_{1}(-I) \cdot \vec{r}_{I C D o r b} \equiv R_{i}^{e} \cdot \vec{r}_{I C D o r b}
$$


where $R_{i}^{e}$ is defined as the rotation matrix from the ICDorb to the ECEF. According to ICD-GPS-200c, the rotation matrix has the following form

$$
R_{i}^{e}=\left(\begin{array}{ccc}
\cos \Omega_{c} & -\sin \Omega_{c} \cos I & \sin \Omega_{c} \sin I \\
\sin \Omega_{c} & \cos \Omega_{c} \cos I & -\cos \Omega_{c} \sin I \\
0 & \sin I & \cos I
\end{array}\right)
$$

where $\Omega_{c}$ is the corrected right ascension of the ascending node, calculated by:

$$
\Omega_{c}=\Omega_{0}+\left(\dot{\Omega}-\dot{\Omega}_{e}\right) t_{k}+\dot{\Omega}_{e} \cdot t_{o e}
$$

where: $\Omega_{0}$ is the right ascension of the ascending node at reference time; $\dot{\Omega}$ is the change rate of the right ascension; $\dot{\Omega}_{e}$ is the angular change rate of the Earth's rotation; $t_{k}$ is the calculation time; and $t_{o e}$ is the reference time of the ephemeris parameters.

To better represent the satellite positions in the orbit, three change rate parameters are used to describe the linear change characteristics of the satellite mean motion $n$, $\Omega_{c}$, and $I$ respectively. These change rate parameters are vitally important for the ECEF satellite velocity and acceleration determination.

The adoption of ICD-GPS-200c orbit representation scheme for use by the GPS Control Segment has some advantages: firstly, the satellite position calculation is more effective given the fact that the GPS orbit is near circular and the effective orbit representation time is short (2 3 hours), there are only two rotations to transform an orbital position into the ECEF system; secondly, there is no need to introduce another parameter, i.e., the change rate of the argument of perigee thus reducing the payload of navigation message. Since perigee itself is hard to be defined in orbits with small eccentricities (Montenbruck and Gill, 2000, p.30), the adopted broadcast orbit representation scheme not only alleviates the navigation payload but also avoids the difficulty in provision of the change rate of the argument of perigee. However, as will be discussed later, it is rather complicated when satellite velocity needs to be determined.

3. TRANSFORM GPS ORBITAL VELOCITY TO ECEF. The transformation of a GPS satellite velocity from the ICDorb system to the ECEF may be simply given through the differentiation of Equation (6) by:

$$
\dot{\vec{r}}_{E C E F}=\dot{R}_{i}^{e} \cdot \vec{r}_{I C D o r b}+R_{i}^{e} \cdot \dot{\vec{r}}_{I C D o r b}
$$

where; $\dot{R}_{i}^{e}$ is the first derivative of the rotation matrix $R_{i}^{e}$ with respect to time; $\dot{\vec{r}}_{I C D o r b}$ is the velocity of the satellite in ICDorb system. These are the two unknowns that must be resolved prior to getting the ECEF velocity.

3.1. Determination of $\dot{R}_{i}^{e}$. The first derivative of the rotation matrix $\dot{R}_{i}^{e}$ with respect to time can be determined from the broadcast ephemeris parameters without any difficulty. The procedures are as follows:

The derivatives of the corrected longitude of ascending node $\Omega_{c}$ and the inclination $I$ are required. These can be done simply through their own definitions by:

$$
\begin{gathered}
\dot{\Omega}_{c}=\dot{\Omega}-\dot{\Omega}_{e} \\
\dot{I}=\dot{i}
\end{gathered}
$$


where $i$ is IDOT, the inclination change rate that is one of the parameters in the broadcast ephemeris.

Differentiating Equation (7) with respect to time, and then substituting the above rates, we have

$\dot{R}_{i}^{e}=\left(\begin{array}{ccc}-\sin \Omega_{c} \cdot \dot{\Omega}_{c} & -\cos \Omega_{c} \cos I \cdot \dot{\Omega}_{c}+\sin \Omega_{c} \sin I \cdot \dot{I} & \cos \Omega_{c} \sin I \cdot \dot{\Omega}_{c}+\sin \Omega_{c} \cos I \cdot \dot{I} \\ \cos \Omega_{c} \cdot \dot{\Omega}_{c} & -\sin \Omega_{c} \cos I \cdot \dot{\Omega}_{c}-\cos \Omega_{c} \sin I \cdot \dot{I} & \sin \Omega_{c} \sin I \cdot \dot{\Omega}_{c}-\cos \Omega_{c} \cos I \cdot \dot{I} \\ 0 & \cos I \cdot \dot{I} & -\sin I \cdot \dot{I}\end{array}\right)$

The magnitude of $\dot{I}$ is in the order of $10^{-10}$ to $10^{-12}$ radians (cf. Gurtner, 2001) while the magnitude of the $\sin I$ and $\cos I$ terms are less than 1.0, so Equation (11) can be simplified by neglecting those $\dot{I}$ terms without losing numerical precision to become

$$
\dot{R}_{i}^{e}=\left(\begin{array}{ccc}
-\sin \Omega_{c} \cdot \dot{\Omega}_{c} & -\cos \Omega_{c} \cos I \cdot \dot{\Omega}_{c} & \cos \Omega_{c} \sin I \cdot \dot{\Omega}_{c} \\
\cos \Omega_{c} \cdot \dot{\Omega}_{c} & -\sin \Omega_{c} \cos I \cdot \dot{\Omega}_{c} & \sin \Omega_{c} \sin I \cdot \dot{\Omega}_{c} \\
0 & 0 & 0
\end{array}\right)
$$

3.2. Determination of $\dot{\vec{r}}_{I C D o r b}$. The satellite coordinate in ICDorb system is calculated by

$$
\vec{r}_{I C D o r b}=\left(\begin{array}{c}
X_{I C D o r b} \\
Y_{I C D o r b}
\end{array}\right)=\left(\begin{array}{c}
r_{c} \cos V c \\
r_{c} \sin V c
\end{array}\right)
$$

where $r_{c}$ is the corrected radius and $V_{c}$ is the corrected argument of latitude respectively. Note that the subscript $c$ is used to indicate that they are "corrected" and calculated relative to the ascending node. These two variables can be calculated as:

$$
\begin{gathered}
r_{c}=a(1-e \cos E)+d r \\
V_{c}=U+\omega+d U
\end{gathered}
$$

where $d r, d U$ are the harmonic perturbation corrections given by:

$$
\begin{aligned}
& d r=c r c \cdot \cos 2(U+\omega)+c r s \cdot \sin 2(U+\omega) \\
& d U=c u c \cdot \cos 2(U+\omega)+c u s \cdot \sin 2(U+\omega)
\end{aligned}
$$

where $\mathrm{crc}, \mathrm{crs}, \mathrm{cuc}$, cus are the harmonic perturbation parameters in the broadcast ephemeris for $r_{c}$ and $V_{c}$ respectively. It is evident from the above equations that the ICDorb system has the positive $\mathrm{x}$-axis passing through the ascending node and the broadcast ephemeris is parameterized accordingly.

The orbital velocity can be obtained by differentiating Equation (13) with respect to time

$$
\dot{\vec{r}}_{I C D o r b}=\left(\begin{array}{c}
\dot{X}_{I C D o r b} \\
\dot{Y}_{I C D o r b}
\end{array}\right)=\left(\begin{array}{l}
\dot{r}_{c} \cos V_{c}-r_{c} \sin V_{c} \cdot \dot{V}_{c} \\
\dot{r}_{c} \sin V_{c}+r_{c} \cos V_{c} \cdot \dot{V}_{c}
\end{array}\right)
$$

Equation (18) shows that the derivatives of $r_{c}$ and $V_{c}$ should be determined prior to the calculation of satellite velocity in the ICDorb coordinate system. 
3.2.1. $\dot{r}_{c}$ Derivation. $\dot{r}_{c}$ may be derived by differentiating Equation (14) with respect to time. The differentiation could be carried out in two steps. The first step is to differentiate the first term in the right hand side of (14) as:

$$
[a(1-e \cdot \cos E)]^{\prime}=a \cdot e \cdot \sin E \cdot \dot{E}
$$

where the superscript prime (') is a differentiation operator and $\dot{E}$ may be obtained from Kepler's equation (Marshall, 2002) or simply by comparison of Equations (1) and (2)

$$
\dot{E}=\frac{n}{1-e \cdot \cos E}
$$

It can also be numerically computed along with the solution of Kepler's equation in an alternative form as:

$$
\dot{M}=\dot{E} \cdot(1-e \cdot \cos E) \Rightarrow \dot{E}=\frac{\dot{M}}{1-e \cdot \cos E}
$$

The second step is to get the derivative of the second term, $d r$, in the right hand side of Equation (14) by differentiating Equation (16), and treating $\omega$ as a constant:

$$
[d r]^{\prime}=-2[c r c \cdot \sin 2(U+\omega)-c r s \cdot \cos 2(U+\omega)] \dot{U}
$$

where the change rate of the true anomaly $\dot{U}$, is still unknown. To comply with Kepler's second law (the radius vector sweeps out equal areas in equal time)

$$
r_{c}^{2} \dot{U} \cdot d t=a \sqrt{1-e^{2}} \cdot n \cdot d t
$$

leads to:

$$
\dot{U}=\frac{a \sqrt{1-e^{2}} \cdot n}{r_{c}^{2}}
$$

3.2.2. $\dot{V}_{c}$ Derivation. $\dot{V}_{c}$, the derivative of the argument of latitude with respect to time, can be derived in a similar manner by differentiating Equation (15) and holding $\omega$ as a constant once again:

$$
\dot{V}_{c}=\dot{U}+[d U]^{\prime}
$$

where the only unknown quantity is the second term in the right hand side. This may be easily obtained by differentiating Equation (17), treating $\omega$ as a constant:

$$
[d U]^{\prime}=-2[c u c \cdot \sin 2(U+\omega)-c u s \cdot \cos 2(U+\omega)] \dot{U}
$$

3.2.3. Implementation program. A $\mathrm{C}++$ implementation of the above velocity algorithm can be found in the source code skyplot.cpp from the National Geodetic Service (NGS) website (Marshall, 2002). It is embedded in function bccalc(...) in the program file of skyplot.cpp. Recently, an independent program using the same algorithm has been presented by Remondi (2004) at the NGS website. The velocity algorithm is described in detail in this paper as derivation of satellite ECEF acceleration requires some of the equations in the velocity determination. 
Table 1. Acceleration and velocity from the NGS SP3 precise positions and velocities (PRN07, 20 August 2002. Accelerations are obtained using the first-order central difference of a Taylor series approximation of SP3 velocities).

\begin{tabular}{lcccccc}
\hline Time & $\mathrm{A}_{\mathrm{x}}\left(\mathrm{m} / \mathrm{s}^{2}\right)$ & $\mathrm{A}_{\mathrm{y}}\left(\mathrm{m} / \mathrm{s}^{2}\right)$ & $\mathrm{A}_{\mathrm{z}}\left(\mathrm{m} / \mathrm{s}^{2}\right)$ & $\mathrm{V}_{\mathrm{x}}(\mathrm{m} / \mathrm{s})$ & $\mathrm{V}_{\mathrm{y}}(\mathrm{m} / \mathrm{s})$ & $\mathrm{V}_{\mathrm{z}}(\mathrm{m} / \mathrm{s})$ \\
\hline $22: 19: 01$ & $0 \cdot 2055$ & $-0 \cdot 3022$ & $0 \cdot 1413$ & $329 \cdot 9513$ & $-888 \cdot 5965$ & $-2997 \cdot 0555$ \\
$22: 19: 02$ & $0 \cdot 2054$ & $-0 \cdot 3022$ & $0 \cdot 1414$ & $330 \cdot 1568$ & $-888 \cdot 8987$ & $-2996 \cdot 9141$ \\
$22: 19: 03$ & $0 \cdot 2054$ & $-0 \cdot 3022$ & $0 \cdot 1414$ & $330 \cdot 3622$ & $-889 \cdot 2009$ & $-2996 \cdot 7727$ \\
$22: 19: 04$ & $0 \cdot 2053$ & $-0 \cdot 3023$ & $0 \cdot 1415$ & $330 \cdot 5676$ & $-889 \cdot 5032$ & $-2996 \cdot 6312$ \\
$22: 19: 05$ & $0 \cdot 2053$ & $-0 \cdot 3023$ & $0 \cdot 1415$ & $330 \cdot 7729$ & $-889 \cdot 8055$ & $-2996 \cdot 4897$ \\
$22: 19: 06$ & $0 \cdot 2052$ & $-0 \cdot 3023$ & $0 \cdot 1416$ & $330 \cdot 9782$ & $-890 \cdot 1078$ & $-2996 \cdot 3481$ \\
$22: 19: 07$ & $0 \cdot 2052$ & $-0 \cdot 3023$ & $0 \cdot 1417$ & $331 \cdot 1834$ & $-890 \cdot 4101$ & $-2996 \cdot 2064$ \\
$22: 19: 08$ & $0 \cdot 2051$ & $-0 \cdot 3023$ & $0 \cdot 1418$ & $331 \cdot 3886$ & $-890 \cdot 7124$ & $-2996 \cdot 0646$ \\
$22: 19: 09$ & $0 \cdot 2051$ & $-0 \cdot 3024$ & $0 \cdot 1418$ & $331 \cdot 5937$ & $-891 \cdot 0147$ & $-2995 \cdot 9228$ \\
$22: 19: 10$ & $0 \cdot 2050$ & $-0 \cdot 3024$ & $0 \cdot 1419$ & 331.7988 & $-891 \cdot 3171$ & $-2995 \cdot 7809$
\end{tabular}

Table 2. Acceleration and velocity from the rotation method using the broadcast ephemeris.

(PRN07, 20 August 2002)

\begin{tabular}{|c|c|c|c|c|c|c|}
\hline Time & $\mathrm{A}_{\mathrm{x}}\left(\mathrm{m} / \mathrm{s}^{2}\right)$ & $\mathrm{A}_{\mathrm{y}}\left(\mathrm{m} / \mathrm{s}^{2}\right)$ & $\mathrm{A}_{\mathrm{z}}\left(\mathrm{m} / \mathrm{s}^{2}\right)$ & $\mathrm{V}_{\mathrm{x}}(\mathrm{m} / \mathrm{s})$ & $\mathrm{V}_{\mathrm{y}}(\mathrm{m} / \mathrm{s})$ & $\mathrm{V}_{\mathrm{z}}(\mathrm{m} / \mathrm{s})$ \\
\hline 22:19:01 & $0 \cdot 2055$ & $-0 \cdot 3022$ & $0 \cdot 1412$ & 329.9518 & -888.5962 & $-2997 \cdot 0552$ \\
\hline $22: 19: 02$ & $0 \cdot 2054$ & $-0 \cdot 3022$ & $0 \cdot 1413$ & $330 \cdot 1573$ & -888.8985 & $-2996 \cdot 9138$ \\
\hline 22:19:03 & $0 \cdot 2054$ & $-0 \cdot 3022$ & $0 \cdot 1413$ & $330 \cdot 3627$ & $-889 \cdot 2007$ & $-2996 \cdot 7724$ \\
\hline $22: 19: 04$ & $0 \cdot 2053$ & $-0 \cdot 3022$ & $0 \cdot 1414$ & $330 \cdot 5680$ & $-889 \cdot 5030$ & -2996.6309 \\
\hline 22:19:05 & $0 \cdot 2053$ & $-0 \cdot 3022$ & $0 \cdot 1415$ & $330 \cdot 7733$ & $-889 \cdot 8052$ & $-2996 \cdot 4894$ \\
\hline 22:19:06 & $0 \cdot 2052$ & $-0 \cdot 3022$ & $0 \cdot 1415$ & $330 \cdot 9786$ & $-890 \cdot 1075$ & $-2996 \cdot 3478$ \\
\hline 22:19:07 & $0 \cdot 2052$ & $-0 \cdot 3023$ & $0 \cdot 1416$ & $331 \cdot 1838$ & $-890 \cdot 4098$ & $-2996 \cdot 2061$ \\
\hline 22:19:08 & $0 \cdot 2051$ & $-0 \cdot 3023$ & $0 \cdot 1417$ & $331 \cdot 3890$ & $-890 \cdot 7121$ & -2996.0643 \\
\hline 22:19:09 & $0 \cdot 2051$ & $-0 \cdot 3023$ & $0 \cdot 1417$ & $331 \cdot 5941$ & $-891 \cdot 0145$ & -2995.9225 \\
\hline $22: 19: 10$ & $0 \cdot 2050$ & $-0 \cdot 3023$ & $0 \cdot 1418$ & 331.7992 & $-891 \cdot 3168$ & -2995.7807 \\
\hline
\end{tabular}

The computation of the GPS satellite velocity in the ICDorb system is "complicated" as can be seen by inspection of the source codes of skyplot.cpp. The complexity is caused mainly by the method of determining change rates of the corrected radius and the corrected argument of latitude, which is due to the ICD-GPS-200's orbital system and its effects on the representation of harmonic perturbation parameters. The process of calculating $\dot{E}$ also contributes to the complexity of the algorithm. Note that treating $\omega$ as a constant at several steps of the derivation process may cause an error in the resultant ICDorb velocity.

Tables 1 and 2 list both velocity and acceleration (for PRN 07 on 20 August 2002) derived using the NGS SP3 precise ephemeris and the rotation method respectively. It is useful to point out that, although the precise ephemeris is defined with reference to the mass centre of a satellite, which differs from the antenna centre to which the broadcast ephemeris is referenced, the velocities can be directly compared with each other. It can be seen from Tables 1 and 2 that although there are small biases in each axis, the velocities derived from the broadcast ephemeris agree with the precise ephemeris within $\pm 1 \mathrm{~mm} / \mathrm{s}$ in each axis.

4. TRANSFORM ORBITAL ACCELERATION TO ECEF. Since the orbital acceleration formula is expressed as a function of the position vector, which 


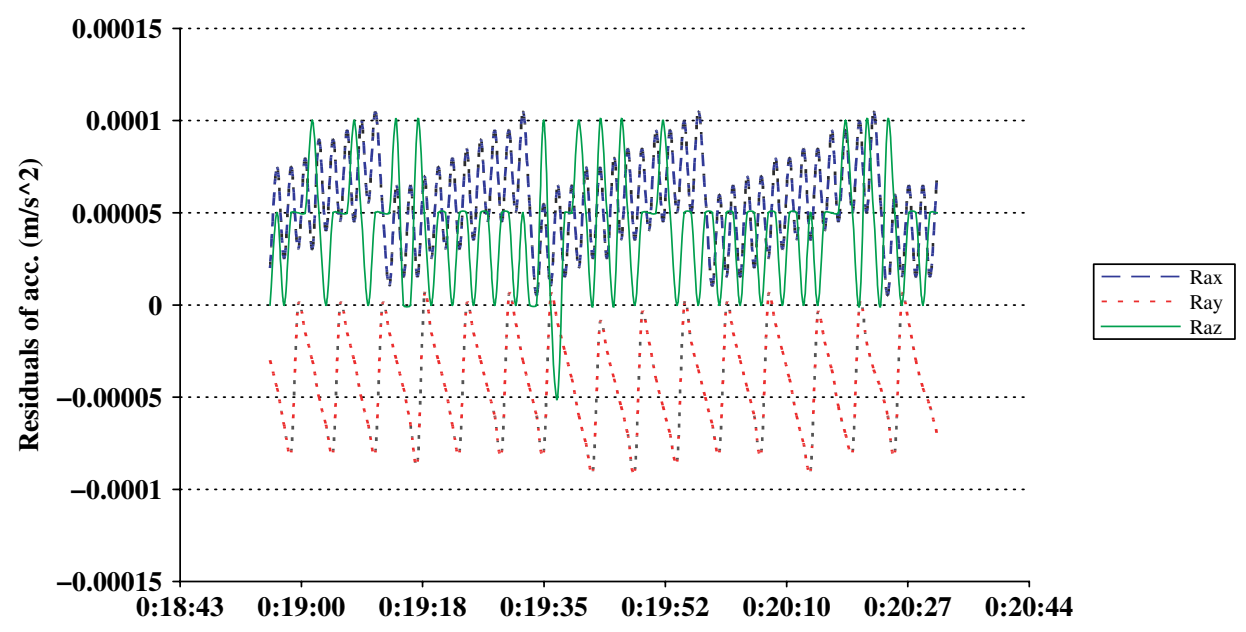

Figure 3. Residuals of the accelerations obtained from the closed-form formula and the precise ephemeris SP3 velocities using the first-order central difference of a Taylor series approximation $(\mathrm{PRN}=07,22: 18: 56 \sim 22: 20: 32,08 / 20 / 2002)$.

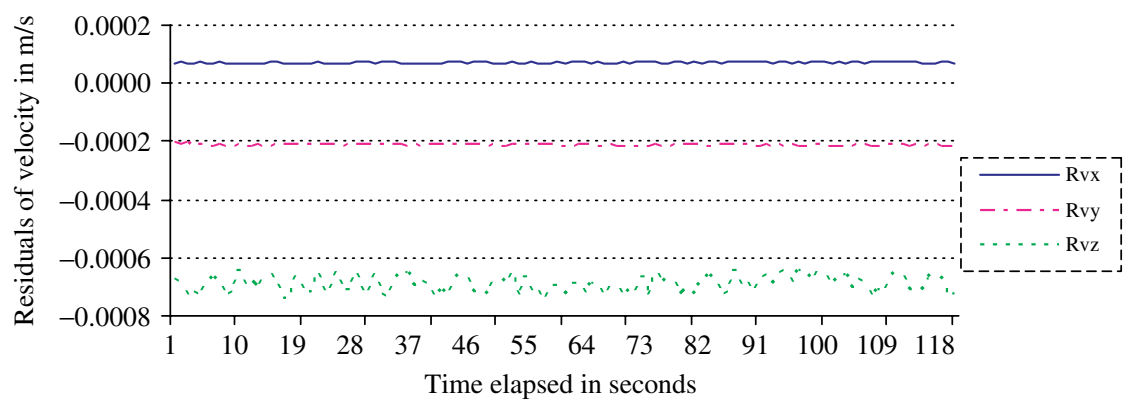

Figure 4. Residuals of the position-differenced ECEF satellite velocities compared with the velocities from the SP3 precise ephemeris (PRN=07, 22:19:00 22:21:00, 08/20/2002).

is independent of the orbital plane orientation, the transformation is much easier, see Equation (3). One only needs to account for the second derivative of the rotation matrix $R_{i}^{e}$ with respect to time, and then the acceleration transformation can be carried out through differentiating Equation (9) with respect to time. This leads to:

$$
\ddot{\vec{r}}_{E C E F}=\ddot{R}_{i}^{e} \cdot \vec{r}_{I C D o r b}+2 \dot{R}_{i}^{e} \cdot \dot{\vec{r}}_{I C D o r b}+R_{i}^{e} \cdot \ddot{\vec{r}}_{I C D o r b}
$$

where $\ddot{R}_{i}^{e}$ is the second derivative of the rotation matrix $R_{i}^{e}$. Taking $\ddot{I}$ and those $\dot{I}$ terms as zero, and $\ddot{\Omega}, \ddot{\Omega}_{e}$ and therefore $\ddot{\Omega}_{c}$ as zero as well, the second derivative of the rotation matrix can be derived from Equation (11) as:

$$
\ddot{R}_{i}^{e}=\left(\begin{array}{ccc}
-\cos \Omega_{c} \cdot \dot{\Omega}_{c}{ }^{2} & \sin \Omega_{c} \cdot \dot{\Omega}_{c}{ }^{2} \cdot \cos I & -\sin \Omega_{c} \cdot \dot{\Omega}_{c}{ }^{2} \cdot \sin I \\
-\sin \Omega_{c} \cdot \dot{\Omega}_{c}{ }^{2} & -\cos \Omega_{c} \cdot \dot{\Omega}_{c}{ }^{2} \cdot \cos I & \cos \Omega_{c} \cdot \dot{\Omega}_{c}{ }^{2} \cdot \sin I \\
0 \cdot 0 & 0 \cdot 0 & 0 \cdot 0
\end{array}\right)
$$


In Equation (27), the satellite position vector $\vec{r}_{I C D o r b}$ is readily derived by Equation (13); $R_{i}^{e}$ and $\dot{R}_{i}^{e}$ are given by Equations (7) and (12) respectively; $\dot{\vec{r}}_{I C D o r b}$ can be calculated from Equation (18), and the ICDorb acceleration can be obtained in terms of $\dot{\vec{r}}_{I C D o r b}$ by Equation (3). Since the perturbations of the satellite orbit have been accounted for by the harmonic correction terms provided by the broadcast ephemeris, Equation (27) is capable of delivering accurate GPS satellite accelerations.

The acceleration from the IGS precise ephemeris is extremely accurate and can be accepted as a true quantity. The accelerations obtained using Equation (27) from the broadcast ephemeris have better than $\pm 0 \cdot 1 \mathrm{~mm} / \mathrm{s}^{2}$ per axis accuracy when compared with the accelerations from the SP3 precise ephemeris, which is evidenced by Figure 3 (Table 1 and Table 2 as well). Thus the derived formula can be confidently used in real-time. This overcomes the concerns raised by Kennedy (2003) about the accuracy of real-time precise acceleration determination applications using GPS due to the accuracy limitations of the broadcast ephemeris. It is understandable that even the broadcast ephemeris is capable of achieving such a high acceleration accuracy since physically, the satellite movement in the orbit is highly stable; and mathematically the large radius of GPS orbit in the denominator of Equation (3) can greatly suppress the error propagation.

5. ALternatives TO GET ECEF SATELlite VELOCITY AND ACCELERATION. It is well known that ECEF satellite positions from the broadcast ephemeris have an accuracy of $\pm 1 \mathrm{~m} \sim 5 \mathrm{~m}$. Due to error propagation, the straightforward method of differentiating the ECEF positions to get the satellite ECEF velocity may lead to a large error. The resultant velocity could be very noisy because of the amplification of the differentiation process. However, due to the complexity of the velocity algorithm, the position method is tested using the first-order central difference of a Taylor series approximation:

$$
\dot{\vec{r}}_{E C E F}(t)=\frac{\vec{r}_{E C E F}(t+\Delta t)-\vec{r}_{E C E F}(t-\Delta t)}{2 \Delta t}
$$

where $t$ is the time of the calculation epoch, $\Delta t$ is the time interval, which is set as one second. Unexpectedly the results are close to the precise velocity of IGS SP3 ephemeris by better than $\pm 1.0 \mathrm{~mm}$ per second per axis, this can be reflected by Figure 4 which shows the performance of this position differencing method. Once again the differential scenario even works well in the satellite orbit!

The promising results of the ECEF satellite velocity obtained through differentiation are explained by the realisation that the errors associated with the orbit positions are not Gaussian white. Potential systematic bias and correlated errors of the satellite ECEF positions due to the errors in the broadcast ephemeris have been significantly mitigated through the differentiation process.

Given the excellent performance of the position-differentiation method for ECEF velocity determination, it is sensible to test the performance of the differential method for acceleration determination, i.e.

$$
\ddot{\vec{r}}_{E C E F}(t)=\frac{\dot{\vec{r}}_{E C E F}(t+\Delta t)-\dot{\vec{r}}_{E C E F}(t-\Delta t)}{2 \Delta t}
$$


where $\Delta t$ is set to one second again. Figure 5 illustrates that the performance of the differential procedure is very good. However, the results are inferior in performance when compared with the accelerations derived from the closed-form formula.

The differentiator used here is a simple Finite Impulse Response (FIR) filter. The frequency response of the FIR differentiator is shown in Figure 6. It approximates the ideal differentiator in lower frequencies which better suits the GPS satellite dynamics in its high orbit. Since even an ideal differentiator has a fixed phase delay, the central difference gives the velocity exactly at the desired epoch. Setting $\Delta t$ to one second gives \pm 0.5 as the filter coefficient, which can further suppress the errors associated with the two positions.

Thus a practical alternative of satellite ECEF position, velocity and acceleration determination algorithm from the broadcast ephemeris is proposed as follows:

- to get positions of $\vec{r}_{E C E F}(t)$ and $\vec{r}_{E C E F}(t \pm \Delta t)$ by using the ICD-GPS-200c algorithm;

- to calculate velocity by using the position differentiation method, see Equation (29); and

- to determine acceleration by using the closed-form rotation formula of Equation (27).

Since the GPS orbit is relatively stable and predictable, polynomial techniques have been widely used to interpolate GPS orbital positions in real-time applications. The polynomial interpolation can greatly accelerate the calculation process of satellite positions. The success of the position differential method implies that, when a polynomial interpolation is used to represent satellite positions, the precise satellite ECEF velocity can be directly obtained through numerical differentiation of the polynomial coefficients. This could contribute to those applications where precise velocities are required at high output rates in real-time. VBOX (2004) is a typical example of such applications whereby positions and velocities of a car are output at a rate of $100 \mathrm{~Hz}$ to facilitate the automobile breaking tests.

6. CONCLUSIONS. GPS satellite orbit system defined by the ICD-GPS-200 differs from the natural satellite orbit system. This difference affects the orbital parameterisation and the algorithms to transform orbital positions to ECEF system. Although the way of broadcast parameterisation has advantages in ECEF satellite position calculation, it is complicated in the determination of ECEF satellite velocity.

This paper proposed an alternative method using a simple differentiator to derive ECEF satellite velocity from the ECEF satellite positions that are calculated using the standard ICD-GPS-200 algorithm. It is demonstrated that $\pm 1 \mathrm{~mm} / \mathrm{s}$ per axis ECEF satellite velocity is achievable by using the first-order central difference of a Taylor series approximation. A closed-form formula is also derived for the determination of GPS satellite ECEF acceleration using the broadcast ephemeris. This formula is capable of achieving an accuracy of better than $\pm 0 \cdot 1 \mathrm{~mm}$ per second squared in each axis. With such a high accuracy of satellite acceleration in real-time, it is possible to detect the line-of-sight range acceleration accurately and therefore to use a GPS receiver as a precise accelerometer. 


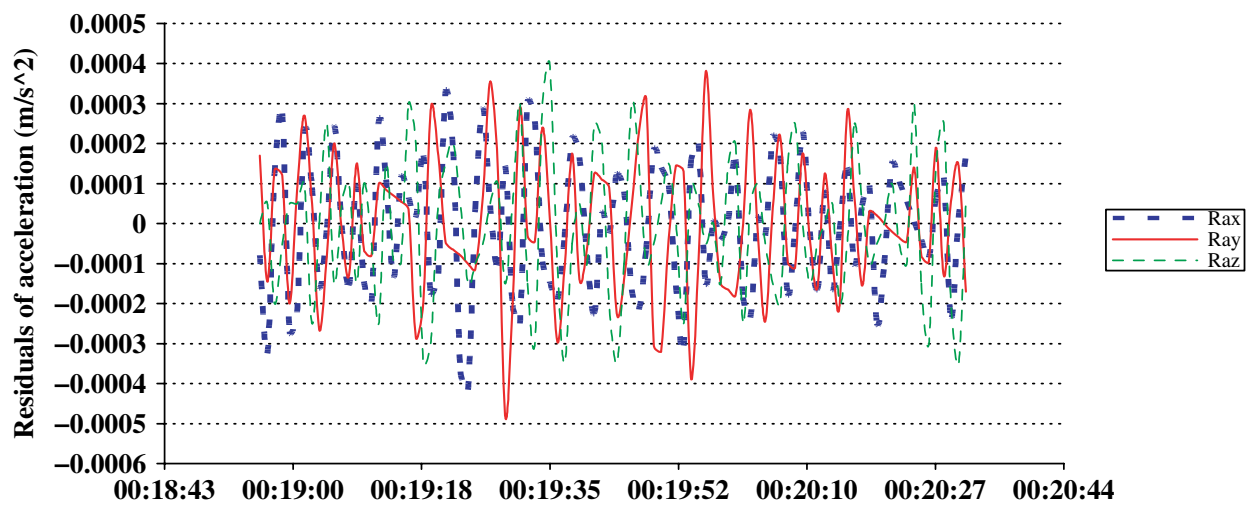

Figure 5. Residuals of the position-differenced ECEF satellite accelerations and the accelerations obtained from the precise SP3 velocities using the first-order central difference of a Taylor series approximation ( $\mathrm{PRN}=07,22: 18: 56 \sim 22: 20: 32,08 / 20 / 2002)$.

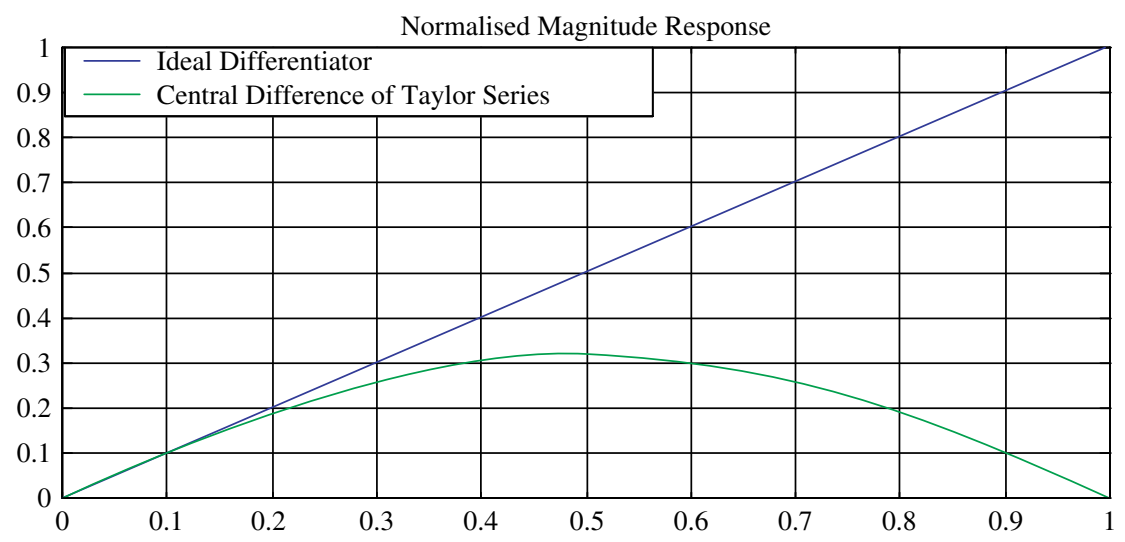

Figure 6. Frequency response of the differentiator of the first-order central difference of a Taylor series approximation.

The proposed algorithm on the GPS satellite ECEF velocity determination simplifies the velocity transformation procedure, and therefore provides a good alternative. The success of the position differential method implies that real-time satellite ECEF velocities can be directly derived through numerical differentiation of the position polynomials. This is favourable for real-time GPS velocity determination applications which require high output rate results. The derived closed-form formula for GPS satellite ECEF acceleration would benefit those who attempt to use a GPS receiver as an accurate accelerometer in real-time.

\section{ACKNOWLEDGEMENT}

The research described in this paper was partially supported by the Cooperative Research Centre (CRC) for microTechnology, Australia. 


\section{REFERENCES}

ARINC (2000) Interface Control Document, Navstar GPS Space Segment/Navigation User Interfaces, ICDGPS-200, Revision C, Arinc Research Corporation, IRN-200C-004, http://www.navcen.uscg.gov/gps/ geninfo/ICD-GPS-200C\%20with\%20IRNs\%2012345.pdf.

Beutler, G. (1998) In GPS for Geodesy, (Eds, Teunissen, P. J. G. and Kleusberg, A.) Springer-Verlag Berlin Heidelberg, Berlin, Germany, 43-110.

Farrell, J. A. and Barth, M. (1999) The Global Positioning System and Inertial Navigation, McGraw-Hill, 0-07-022045-X.

Gurtner, W. (2001) RINEX: the Receiver Independent Exchange Format Version 2.10, http:// www.ngs.noaa.gov/CORS/Rinex2.html, Accessed on 9 Aug 2004.

Hofmann-Wellenhof, B., Lichtenegger, H. and Collins, J. (2001) Global Positioning System Theory and Practice, Springer-Verlag/Wien New York, 3-211-83534-2.

Jekeli, C. (1994) On the Computation of Vehicle Accelerations Using GPS Phase Accelerations, International Symposium on Kinematic Systems in Geodesy, Geomatics and Navigation, Banff, Canada. 473-481.

Jekeli, C. and Garcia., R. (1997) Journal of Geodesy, 71 (10), 630-639.

Kennedy, S. L. (2003) Navigation, Journal of the Institute of Navigation, 50 (1), 9-19.

Kleusberg, A., Peyton, D. and Wells, D. (1990) Airbornegravimetry and the Global Positioning System, IEEE 1990 273-278.

Marshall, J. (2002) GPS solutions, 6 (1-2), 118-120.

Misra, P. and Enge, P. (2001) Global Positioning System: Signals, Measurements, and Performance, GangaJamuna Press, Lincoln, Massachusetts. 0-9709544-0-9.

Montenbruck, O. and Gill, E. (2000) Satellite Orbits: Models, Methods, and Applications, Springer Verlag, Heidelberg. 3-540-67280-x.

Remondi, B. W. (2004) GPS solutions, 8 (3), 181-183.

Simsky, A. and Boon, F. (2003) Carrier Phase and Doppler-Based Algorithms for Real-Time Standalone Positioning, Proceedings of GNSS 2003, The European Navigation Conference, Graz, Austria.

Spofford, P. R. and Remondi, B. W. (2004) The National Geodetic Survey Standard GPS Format SP3, http://gibs.leipzig.ifag.de/cgi-bin/sp3_doc.cgi?en, Accessed on Sept. 102004.

VBOX (2004) VBOX II 100Hz Speed Sensor, http://www.m-techautomotive.co.uk/vbox/downloads/ vb2sps100_data.pdf, Accessed on Nov. 252004.

Zhang, J., Zhang, K., Grenfell, R., Li, Y. and Deakin, R. (2004) On Real-time High Precision Velocity Determination for Standalone GPS Users, The International Symposium on GPS/GNSS,2004, Sydney, December 6-8 2004. Paper(66).

Zhang, K., Grenfell, R., Deakin, R., Li, Y. and Zhang, J. (2003) Current Development of a Low-Cost, High Output Rate RTK GPS Multisensor System for Rowers, Proceedings of SatNav 2003, Melbourne, Australia. 
Reproduced with permission of the copyright owner. Further reproduction prohibited without permission. 